\title{
Efficacy and relapse rates of different Isotretinoin dosages in treating acne vulgaris: systemic review
}

\author{
Authors: Tyng Hwey Tan, ${ }^{1}$ Rachel Hallett ${ }^{2}$ and Paul Devakar Yesudian ${ }^{1}$
}

\section{Aims}

Isotretinoin has been used, over the last few decades, to treat moderate to severe acne vulgaris that is resistant to adequate courses of standard therapy with systemic antibacterials and topical therapy. However, the ideal dosing regime remains controversial. The effects of different isotretinoin dosages on relapse rates are unclear. Systematic review of clinical trials has been conducted to examine the ideal isotretinoin regime to achieve complete acne clearance, and relapse rate of acne vulgaris following isotretinoin treatment.

\section{Methods}

Pubmed and EMBASE were searched for clinical trials published between 1980 and April 2015. The International Clinical Trials Registry Platform was also searched for any relevant ongoing clinical trials. The research was restricted to English literature. A single reviewer assessed the eligibility of each study based on the predefined inclusion criteria (ie any patients with acne vulgaris treated with isotretinoin, clinical trials investigating the efficacy and relapse/recurrence/retrial rates of isotretinoin in treating acne vulgaris compared to other active treatment/ placebo/isotretinoin of different doses). The Cochrane Collaboration's tool for assessing risk of bias was used to assess the validity of each included study.

\section{Results}

3,882 abstracts were reviewed and 41 studies were included in the systematic review. The studies were of greatly diverse designs, especially in terms of the characteristics of study population, isotretinoin regimes and outcome measurements. Different acne grading systems were used across studies and this has further increased the difficulty of comparing results between studies. Relapse rate was also defined differently. Generally, the studies have certain degree of risk of bias.

Authors: ${ }^{1}$ Betsi Cadwaladr University Health Board, Bodelwyddan, UK; ${ }^{2}$ Bangor University, Bangor, UK

\section{Conclusions}

In view of great heterogeneity of the studies, it is difficult to produce any recommendation based on the existing evidences. This might be due to heterogeneous nature of acne vulgaris and different expectations of ideal isotretinoin treatment regime. This has also reflected the importance of standard clinical trials protocol items (eg SPIRIT Initiative (Standard Protocol Items: Recommendations for Interventional Trials)) and clinical trials reporting guideline (eg CONSORT (Consolidated Standards of Reporting Trials)). These will not only improve the quality of clinical trials, but will also increase the potential of summarising relevant study results to provide a more precise effect of interest. 